
\title{
25 Research Soure \\ Thyroid Stimulating Hormone Suppression and Recurrence After Thyroid Lobectomy For Papillary Thyroid Carcinoma
}

\section{Mi Rye Bae}

Asan Medical Center

\section{Sung Hoon Nam}

Asan Medical Center

Jong-Lyel Roh ( $\nabla$ jonglyel.roh@gmail.com )

Bundang CHA Medical Center https://orcid.org/0000-0002-1537-1977

\section{Seung-Ho Choi}

Asan Medical Center

Soon Yuhl Nam

Asan Medical Center

\section{Sang Yoon Kim}

Asan Medical Center

\section{Research Article}

Keywords: Papillary thyroid carcinoma, lobectomy, hypothyroidism, recurrence, risk factor.

Posted Date: August 19th, 2021

DOI: https://doi.org/10.21203/rs.3.rs-818671/v1

License: (c) (1) This work is licensed under a Creative Commons Attribution 4.0 International License.

Read Full License

Version of Record: A version of this preprint was published at Endocrine on October 23rd, 2021. See the published version at https://doi.org/10.1007/s12020-021-02911-x. 


\section{Abstract \\ Background}

Thyroid lobectomy is recommended as the primary treatment for low-risk thyroid cancer. However, recurrence and hypothyroidism may develop after lobectomy, necessitating thyroid hormone supplementation. The 2015 American Thyroid Association (ATA) guidelines recommended postlobectomy thyroid-stimulating hormone (TSH) suppression. This study examined the need for TSH suppression and recurrence after lobectomy for unilateral papillary thyroid carcinoma (PTC).

\section{Methods}

This study involved 369 patients who underwent thyroid lobectomy and ipsilateral central neck dissection for PTC between 2007 and 2015. Thyroid function tests were performed before and regularly after lobectomy. Binary logistic regression analyses were used to find factors predictive of the post-lobectomy need for TSH suppression that was defined by the 2015 ATA guidelines.

\section{Results}

Serum TSH concentrations gradually increased after lobectomy: proportions with TSH $>2 \mathrm{mIU} / \mathrm{L}$ at postlobectomy $1,3 \sim 6,12$, and 24 months were found in $77.0 \%, 82.3 \%, 66.7 \%$, and $59.9 \%$, respectively. After lobectomy, 168 (45.5\%) patients received levothyroxine (T4) supplementation. Multivariate logistic regression analyses showed that pre-TSH level $>2 \mathrm{mIU} / \mathrm{L}$ was the sole independent variable predictive of the need for post-lobectomy TSH suppression $(P=0.003)$. During the median follow-up of 72 months, recurrence was found in $4(1.1 \%)$ patients who never received T4 supplementation and had postlobectomy TSH levels $>2 \mathrm{mlU} / \mathrm{L}$.

\section{Conclusions}

Our data show that thyroid lobectomy for unilateral PTC is associated with a low recurrence rate, but a significant risk of hypothyroidism. Preoperative TSH level can predict the need for post-lobectomy TSH suppression compliant with the 2015 ATA guidelines.

\section{Introduction}

Papillary thyroid carcinoma (PTC) is associated with excellent disease-specific survival of more than $90 \%$ $[1,2]$. Risks of survival and recurrence can be affected by various clinical and pathological factors, e.g., age, sex, tumor size and extrathyroidal extension, completeness of resection, distant metastasis, and comorbidity. After a study based on the National Cancer Data Base (NCDB, 1985-1998), Bilimoria and colleagues [3] suggested that total thyroidectomy improved survival and recurrence rates for PTC $\geq 1 \mathrm{~cm}$, 
as compared with lobectomy. In contrast, another analysis based on the NCDB (1998-2006) observed no statistically significant difference in survival between patients who underwent total thyroidectomy and those who underwent lobectomy [4]. A further study also showed excellent outcomes, including a $95.2 \%$ cause-specific survival rate at 25 years after thyroid lobectomy for PTC [5]. The 2015 American Thyroid Association (ATA) guideline recommended that lobectomy can be applied to thyroid cancer $>1 \mathrm{~cm}$ and $<4$ $\mathrm{cm}$ in size, without extrathyroidal extension or clinical lymph node (LN) metastasis [6]. However, the recurrence and survival associated with using total thyroidectomy versus lobectomy as the initial optimal surgical extent for small unilateral PTC are still debated [7].

High thresholds are increasingly incorporated into the guidelines for thyroid cancer surgery, particularly for low-risk disease [8], possibly with a view to the avoidance of potential complications and costeffectiveness $[9,10]$. Thyroid lobectomy is commonly associated with less surgical morbidity than total thyroidectomy, which might influence patients' quality of life [11]. Postoperative hypocalcemia, voice and throat dysfunction, and other complication rates are markedly more often observed in patients who have undergone total thyroidectomy $[11,12]$. The complication rates after lobectomy are commonly low, but increased thyroid-stimulating hormone $(\mathrm{TSH})$ levels $(>2 \mathrm{mIU} / \mathrm{L}$ ) are observed in most patients who undergo lobectomy [13]. A high rate (55.8\%) of post-lobectomy hypothyroidism can occur along with several risk factors, e.g., age and preoperative TSH level [14].

The previous American Thyroid Association (ATA) guidelines recommended that TSH level after lobectomy remains biochemically euthyroid ( $\mathrm{TSH}<5 \mathrm{mlU} / \mathrm{L})$ [15]. The 2015 ATA guidelines made the revised recommendation that TSH may be maintained in the range of $0.5-2 \mathrm{mIU} / \mathrm{L}$ during post-lobectomy surveillance [6]. TSH suppression therapy reduces the recurrence of patients who underwent thyroidectomy for differentiated thyroid cancer [16]. Levothyroxine (T4) supplementation for TSH suppression may be prescribed in patients with TSH levels $>2 \mathrm{mIU} / \mathrm{L}$ after thyroid lobectomy $[17,18]$. However, it is still unclear whether post-lobectomy TSH suppression reduces recurrence risk for differentiated thyroid cancer $[19,20]$. Although there have been several studies on the incidence and risk factors of post-lobectomy hypothyroidism, further studies might be required for the analyses of postlobectomy TSH changes and the need for T4 supplementation meeting the criteria of the 2015 ATA guidelines [21], in this era of de-escalation paradigms for differentiated thyroid cancer. Therefore, we here examined the need for TSH suppression and recurrence after thyroid lobectomy for unilateral PTC.

\section{Materials And Methods}

\section{Study patients}

We retrospectively analyzed the patients who underwent thyroid lobectomy at our department of tertiary referral center between 2007 and 2015. Inclusion criteria were the euthyroid patients who underwent thyroid lobectomy for previously untreated PTC localized to a single lobe. All the patients received a preoperative diagnosis by high-resolution ultrasonography (US) and fine-needle aspiration biopsy and were scheduled for thyroid lobectomy. Exclusion criteria were patients who underwent total or subtotal 
thyroidectomy or completion thyroidectomy, had other thyroid malignancies, a prior history of thyroidectomy or previous neck irradiation, preoperative hypo-/hyperthyroidism or T4 or anti-thyroid medications, and inadequate information without a regular follow-up and surveillance of thyroid function tests for at least 2 years. This study was approved by the Institutional Review Board and the need for obtaining written informed consent from each patient was waived.

\section{Treatments and follow-up}

According to our institutional policy, all the study patients underwent thyroid lobectomy plus central compartment neck dissection (CND) involving pretracheal, prelaryngeal, and ipsilateral paratracheal LN [6], even when there was no evidence of clinical LN metastasis [22]. The primary and nodal samples were sent for pathological examination of tumor multifocality, extrathyroidal extension, lymphovascular invasion, and lymphocytic thyroiditis. All the CND samples from each patient were also carefully examined to identify indications of nodal metastasis in the central neck compartment.

All the postoperative complications were reported and reviewed. No patients received radioactive iodine (RAl) ablation therapy. After surgery, the patients underwent regular follow-up involving clinical examination, neck US, chest radiography, and assessment of serum thyroid function. The patients were scheduled to visit the outpatient clinic at the $1 \mathrm{st}, 3 \mathrm{rd}-6 \mathrm{th}$, and 12th months postoperatively, and annually thereafter. Any lesions suspicious of recurrence were confirmed with US-guided fine-needle aspiration biopsy and patients with loco-regional recurrence were scheduled for re-operation.

\section{Definition of thyroid function status}

According to the guidelines for diagnosis and management of subclinical thyroid disease and the standard practice of our institutional laboratory, euthyroidism was defined as the presence of normal levels of serum TSH (0.4-5.0 mIU/L) and free L-thyroxine (fT4, 0.8-1.9 ng/dL). Hypothyroidism was defined as the elevation of serum TSH levels beyond the upper limit of the reference range $(>5.0 \mathrm{mIU} / \mathrm{L})$ [23]. All study patients underwent regular assessments of serum TSH and T4 levels preoperatively (baseline) and at every postoperative visit at the outpatient clinic. Patients with subclinical hypothyroidism, with TSH increase to $>10 \mathrm{mIU} / \mathrm{L}$, or overt hypothyroidism were assigned T4 replacement [14]. T4 replacement was not prescribed for patients with euthyroidism or subclinical hypothyroidism, without clinical symptoms/signs [14]. Serum TSH and fT4 were calculated for patients who were not on T4 supplementation at the time that thyroid function tests were obtained [21]. To identify factors predictive of the need for T4 supplementation meeting the criteria of the 2015 ATA guidelines [6], the TSH levels were defined at $2.0 \mathrm{mIU} / \mathrm{L}$ : a group with $\mathrm{TSH} \leq 2.0 \mathrm{mIU} / \mathrm{L}$ without T4 supplementation and another group with TSH > $2.0 \mathrm{mIU} / \mathrm{L}$ regardless of T4 supplementation at post-lobectomy 1 year [21, 24].

\section{Statistical analysis}

Median and interquartile range (IQR) are used to summarise continuous variables and number and percentage are used for descriptive data. The end-points of interest were post-lobectomy hypothyroidism and recurrence. Recurrence-free survival (RFS) was defined as the time from surgery to the time of 
recurrence at any site, or the last follow-up. Binary logistic regression analyses were used to analyze the relationship between the post-lobectomy need for TSH suppression and age, sex, tumor and nodal pathological findings, tumor-node-metastasis (TNM) stage (proposed by the American Joint Committee on Cancer, 8th ed.), MACIS score, and the laboratory findings of preoperative thyroid function. The estimated odds ratio (OR) and $95 \% \mathrm{Cl}$ were calculated. Two-sided $P$-values $<0.05$ were considered statistically significant. Statistical analyses were performed using IBM ${ }^{\circledR}$ SPSS ${ }^{\circledR}$ Statistics version 24.0 for Windows (IBM, Armonk, NY, USA).

\section{Results}

\section{Patient characteristics}

This study included a total of 369 lobectomy patients, comprising 89 (24.1\%) men and 280 (75.9\%) women, with a median age of 49 years (IQR, 40-55). Table 1 summarises the demographic and clinicopathological data of patients. Most tumors $(n=323,87.5 \%)$ were micropapillary thyroid carcinomas $\leq 1 \mathrm{~cm}$ in size. Extrathyroidal extension and lymphovascular invasion were found in 114 (30.9\%) and 70 (19.0\%) patients, respectively. Lymphocytic thyroiditis was found in 67 (18.2\%) patients. Pathological TNM staging indicated $349(94.6 \%)$ patients as stage I and $20(5.4 \%)$ patients as stage II. Pathological LN metastasis in the central neck compartment was found in 73 (19.8\%) patients. The median MACIS score was 4.3 and a score $>6.0$ was found in 11 (3.0\%) patients. A few patients had postoperative complications: wound seroma, hematoma, and temporary vocal fold paralysis were found in $2(0.5 \%), 1(0.3 \%)$, and $1(0.3 \%)$ patients, respectively. During the median follow-up period of 72 months (IQR, 56-84 months), only $4(1.1 \%)$ patients had recurrence: $3(0.8 \%)$ patients had a recurrence in the remnant thyroid gland and $1(0.3 \%)$ patient had a recurrence in the lateral neck LNs. One patient died of gallbladder cancer, but the remaining patients survived at the time of the last follow-up. The 5- and 10year RFS rates were $99.5 \%$ and $99.1 \%$, respectively. 
Table 1

Patients characteristics $(N=369)$

\begin{tabular}{|c|c|c|}
\hline Characteristic & $N$ & $\%$ \\
\hline Age, years (IQR) & $49(40-55)$ & \\
\hline$\geq 55$ years & 102 & 27.6 \\
\hline Sex, male/female & $89 / 280$ & $24.1 / 75.9$ \\
\hline Size of tumor (mm), median (IQR) & $7(5-9)$ & \\
\hline$>1 \mathrm{~cm}$ & 46 & 12.5 \\
\hline Multifocality & 35 & 9.5 \\
\hline \multicolumn{3}{|l|}{ Extrathyroidal extension } \\
\hline Microscopic/macroscopic & $98 / 16$ & $26.6 / 4.3$ \\
\hline Lymphovascular invasion & 70 & 19.0 \\
\hline Lymphocytic thyroiditis & 67 & 18.2 \\
\hline \multicolumn{3}{|l|}{ pTNM staging } \\
\hline $\mathrm{T} 1 / \mathrm{T} 2 / \mathrm{T} 3 / \mathrm{T} 4$ & $338 / 15 / 16 / 0$ & $91.6 / 4.1 / 4.3 / 0$ \\
\hline No/N1a & $296 / 73$ & $80.2 / 19.8$ \\
\hline Overall I/II/III/IV & $349 / 20 / 0 / 0$ & $94.6 / 5.4 / 0 / 0$ \\
\hline No. of total LNs harvested, median (IQR) & $6(4-9)$ & \\
\hline No. of LNs involved, $0 / 1 / 2 / 3 / 5$ & $296 / 52 / 15 / 5 / 1$ & $80.2 / 14.1 / 4.1 / 1.4 / 0.3$ \\
\hline MACIS score, median (IQR) & $4.3(3.6-4.8)$ & \\
\hline \multicolumn{3}{|l|}{ Follow up information } \\
\hline Median follow up (IQR), months & $72(56-84)$ & \\
\hline Recurrence & 4 & 1.1 \\
\hline Remnant thyroid gland & 3 & 0.8 \\
\hline Lateral neck LNs & 1 & 0.3 \\
\hline Last status, NED/DOD/DOC/AD & $368 / 0 / 1 / 0$ & $99.7 / 0 / 0.3 / 0$ \\
\hline
\end{tabular}

Abbreviations: AD; alive with disease; DOC, died of other causes; DOD, died of disease (index cancer); IQR, interquartile range; $\mathrm{LN}$, lymph node; MACIS score, distant metastasis, patient agge, completeness of resection, local invasion and tumor size score; NED, no evidence of disease; ND, neck dissection; pTNM, pathological tumor-node-metastasis stage proposed by the American Joint Committee on Cancer (AJCC, 8th ed.). 


\section{Factors predictive of the need for TSH suppression after thyroid lobectomy}

Serum TSH concentrations gradually increased up to $3 \sim 6$ months and then decreased to a level higher than the preoperative level, whereas fT4 concentrations had the opposite trend with TSH in the serial measurements (Fig. 1A). The proportions of patients with $\mathrm{TSH}>2 \mathrm{mIU} / \mathrm{L}$ at post-lobectomy $1,3 \sim 6,12$, and 24 months were found in $77.0 \%, 82.3 \%, 66.7 \%$, and $59.9 \%$, respectively (Fig. 1B). The post-lobectomy proportions of $\mathrm{TSH}>5 \mathrm{mIU} / \mathrm{L}$ at the time points were $28.0 \%, 32.3 \%, 17.3 \%$, and $16.8 \%$, respectively. Of 369 study patients, 168 (45.5\%) received T4 supplementation after lobectomy. At post-lobectomy 1 year, 134 of $201(66.7 \%)$ patients were indicated to receive T4 supplementation according to the 2015 ATA guidelines (Fig. 2). Four patients with post-lobectomy recurrence were not on T4 supplementation and had TSH levels $>2 \mathrm{mIU} / \mathrm{L}$. There were no clinicopathological factors significantly associated with RFS after thyroid lobectomy $(P>0.1)$.

Table 2 shows the relationship between clinicopathological factors and the need for TSH suppression: preoperative TSH level $>2 \mathrm{mIU} / \mathrm{L}$ was the significant factor requiring post-lobectomy $\mathrm{T} 4$ supplementation $(P<0.001)$. Multivariate logistic regression analyses showed that preoperative TSH level $(\mathrm{OR}=2.182,95 \%$ $\mathrm{Cl}, 1.301-3.659 ; P=0.003)$ was the sole independent variable that predicted the post-lobectomy need for TSH suppression (Table 3). 
Table 2

Binary logistic regression analysis of factors predictive of the need for TSH suppression $^{a}$

\begin{tabular}{|llll|}
\hline Characteristic & OR & $95 \%$ Cl & $P$ \\
\hline Age, $\geq 55$ years & 1.232 & $0.731-2.076$ & 0.433 \\
\hline Sex, female/male & 1.623 & $1.975-2.703$ & 0.063 \\
\hline Pathological findings & & & \\
\hline Tumor size, $>1 \mathrm{~cm}$ & 1.311 & $0.549-3.128$ & 0.542 \\
\hline Multifocality & 1.438 & $0.647-3.194$ & 0.373 \\
\hline Extrathyroidal extension & 1.444 & $0.892-2.337$ & 0.135 \\
\hline Lymphovascular invasion & 1.196 & $0.668-2.142$ & 0.546 \\
\hline Central LN involvement & 1.367 & $0.812-2.302$ & 0.240 \\
\hline MACIS, $>6.0$ & 2.047 & $0.580-7.223$ & 0.266 \\
\hline Preoperative laboratory findings & & & \\
\hline Anti-thyroglobulin positivity, $>60 \mathrm{IU} / \mathrm{mL}$ & 1.773 & $0.961-3.272$ & 0.067 \\
\hline Anti-peroxidase positivity, $>60 \mathrm{IU} / \mathrm{mL}$ & 1.803 & $0.811-4.007$ & 0.148 \\
\hline TSH level, > 2 mIU/L & 2.385 & $1.487-3.823$ & $<0.001^{*}$ \\
\hline Lymphocytic thyroiditis & 1.396 & $0.779-2.501$ & 0.263 \\
\hline
\end{tabular}

Abbreviations: $\mathrm{Cl}$, confidence interval; $\mathrm{OR}$, odds ratio; LN, lymph node; MACIS score, distant metastasis, patient age, completeness of resection, local invasion, and tumor size score; TSH, thyroid-stimulating hormone.

${ }^{a}$ According to the 2015 ATA guidelines that recommend the maintenance of TSH in the mid to lower reference range $(0.5-2 \mathrm{mU} / \mathrm{L})$.

${ }^{*} P<0.05$. 
Table 3

Multivariate logistic regression analyses indicating the need for TSH suppression

\begin{tabular}{|llllll|}
\hline Characteristic & $\boldsymbol{\beta}$ & SE & $\operatorname{Exp}(\boldsymbol{\beta})$ & $95 \% \mathrm{Cl}$ & $\boldsymbol{P}$ \\
\hline Sex, female & 0.537 & 0.304 & 1.711 & $0.942-3.106$ & 0.078 \\
\hline Anti-thyroglobulin positivity, $>60 \mathrm{IU} / \mathrm{mL}$ & 0.480 & 0.357 & 1.616 & $0.803-3.254$ & 0.179 \\
\hline Anti-peroxidase positivity, $>60 \mathrm{IU} / \mathrm{mL}$ & 0.475 & 0.454 & 1.607 & $0.661-3.910$ & 0.296 \\
\hline Pre-TSH level, $>2 \mathrm{mIU} / \mathrm{L}$ & 0.780 & 0.264 & 2.182 & $1.301-3.659$ & $0.003^{*}$ \\
\hline Constant & -0.951 & 0.528 & & & \\
\hline
\end{tabular}

Abbreviations: $\mathrm{Cl}$, confidence interval; $\operatorname{Exp}(\beta)$, odds ratio; SE, standard error; Pre-TSH, preoperative thyroidstimulating hormone.

${ }^{*} P<0.05$.

\section{Discussion}

The present study showed that post-lobectomy complications developed rarely after thyroid lobectomy, even when combined with CND. The overall complication rates appeared to be quite low as compared to those after total thyroidectomy [12]. However, post-lobectomy hypothyroidism was a major clinical problem, because a considerable number of patients required thyroid hormone replacement therapy. A previous systematic review reported the pooled risk of post-lobectomy hypothyroidism as $22 \%$ (range, 7-49\%) [25]. Two studies also showed the incidence of subclinical hypothyroidism (defined as TSH level $>4.5 \mathrm{mIU} / \mathrm{L}$ ) after hemithyroidectomy occurred in as many as $55.8 \%$ (226 of 405 patients) and $64.2 \%$ (215 of 335 patients) of post-lobectomy cases $[14,26]$. In most cases $(84.5 \%)$, the condition developed in the early postoperative months (1-3 months postoperatively) [14]. Another study showed that hypothyroidism might develop in $43.3 \%$ ( 145 of 335 patients) within 12 months post-lobectomy and in $20.9 \%$ (70 of 335 patients) thereafter and that 119 of 215 (55.3\%) patients with such hypothyroidism eventually recovered to euthyroid state [26]. A further study also showed that increased TSH levels $>2$ $\mathrm{mIU} / \mathrm{L}$ occurred in 59\% patients within 12 months after thyroid lobectomy [13]. The present study also showed the elevation of serum TSH levels at 1-6 months after lobectomy and the need for T4 supplementation in most patients undergoing thyroid lobectomy.

The 2015 ATA guidelines recommend maintaining the TSH level of 0.5-2 mIU/L after thyroid lobectomy for low-risk thyroid cancer [6]. In the present study, the proportions of patients with the need for T4 supplementation were found $82.3 \%$ at $3 \sim 6$ months and $66.7 \%$ at 1 year after lobectomy. A recent study also showed that a high proportion of 168 study patients met the criteria for T4 supplementation (TSH levels $>2 \mathrm{mlU} / \mathrm{L}$ ) after lobectomy: $67.9 \%$ at 6 weeks with a median $\mathrm{TSH}$ of 3.73 and $76.2 \%$ at $6-12$ months with a median TSH of 3.43 [21]. Preoperative TSH level was a sole significant predictor associated with meeting criteria for TSH suppression $(\mathrm{OR}=1.70,95 \% \mathrm{Cl}=1.06-3.40, P=0.04)$ [21]. The same result was found in the present and previous multiple studies: high preoperative TSH levels were a 
significant risk factor for post-lobectomy hypothyroidism [14, 25-29]. A meta-analysis has shown that other factors associated with increased risk for hypothyroidism are anti-thyroid peroxidase antibodypositivity $(48 \%$ vs. $19 \%, P=0.001)$ and a high degree of thyroid inflammation $(49 \%$ vs. $10 \%, P=0.006)$ [25]. There is still controversy whether TSH suppression therapy increases RFS and overall survival rates in patients undergoing thyroid lobectomy $[19,20]$. Further details on this issue might be skipped. In addition, the T4 supplementation lowering serum TSH level might be balanced with its potential risk of several metabolic complications, e.g., cardiovascular disease and osteoporosis [30, 31]. Further prospective studies are required to elucidate the role of TSH suppression therapy in post-lobectomy patients [18].

The present study showed a very low rate of recurrence after thyroid lobectomy for PTC. During a median follow-up period of 72 months, post-lobectomy recurrence occurred in only $4(1.1 \%)$ patients, with high RFS rates. Adam and colleagues [4] found the overall survival of lobectomy cases similar to those with total thyroidectomy in the patients with PTC $>1 \mathrm{~cm}$, although more $\mathrm{LN}$, extrathyroidal and multifocal diseases were found in patients with total thyroidectomy. Thyroid lobectomy appeared to result in a survival outcome equal to that achieved by total thyroidectomy in stage I PTC patients $<45$ years of age [32]. A previous study also showed the overall low recurrence rate and high cause-specific survival rate after thyroid lobectomy in 1088 PTC patients with a median follow-up of 17.6 years [5]. At 25 years after lobectomy, remnant thyroid, regional LN, and distant site recurrence rates were $93.5 \%, 90.6 \%$, and $93.6 \%$, respectively. They advocated that lobectomy in PTC patients aged $<45$ years and with a tumor size of $\leq 4$ $\mathrm{cm}$ was a valid alternative to total thyroidectomy. Another study also showed excellent outcomes in terms of recurrence: only 23 (3.1\%) of 734 PTC patients with thyroid lobectomy developed recurrent diseases [33]. They found a 10 -year recurrence rate of $0.8 \%$ versus $7.8 \%$ in the cases without and with extrathyroidal extension, respectively. The safety for extended applications of lobectomy, e.g., in PTC with extrathyroidal extension, multifocality, and other adverse findings, as well as tumor size $1.1-4 \mathrm{~cm}$, should be further examined. In our cohort, considerable numbers of patients had adverse pathological findings. The intermediate- or high-risk patients might have an increased probability for post-thyroidectomy recurrence, which appears to be diminished by implementing unilateral CND combined with thyroid lobectomy [22]. However, this should be examined by further studies for the initial optimal extent of surgery in LN dissection as well as thyroidectomy.

Our study had the potential limitations of a retrospective design. A few cases of PTC $>1 \mathrm{~cm}$ were included in our study because the criteria of tumor size for lobectomy strictly followed the previous version of the ATA guidelines [15]. Routine performance of CND in our study patients is not supported by the previous and current ATA guidelines $[6,15]$. However, this approach did allow us to gain accurate pathological nodal information, and other clinical and pathological findings defined considerable numbers of patients with intermediate or high risk in our studies. This information might facilitate further revisions of the current guideline for the extended applications of thyroid lobectomy for differentiated thyroid cancer. Long-term follow-up to obtain accurate information on post-lobectomy recurrence might also be required. 
In conclusion, our data show that thyroid lobectomy for PTC localized to a single lobe is associated with a low recurrence rate, but is significantly associated with post-lobectomy hypothyroidism. After thyroid lobectomy, a considerable number of patients had the elevation of serum TSH concentrations $>2 \mathrm{mIU} / \mathrm{L}$ and the need for TSH suppression to the level of 0.5-2.0 mIU/L. Preoperative TSH level can predict the need for post-lobectomy TSH suppression therapy. This might help identify patients who are likely to require thyroid hormone supplementation.

\section{Declarations}

Acknowledgments: No funding was received in support of this work.

Author Contributions: Prof. Roh had full access to all of the data in the study and take responsibility for the integrity of the data and the accuracy of the data analysis.

Concept and design: Bae, Nam, Roh

Acquisition, analysis, or interpretation of data: Bae, Nam, Roh, Choi, Nam, Kim

Drafting of the manuscript: Bae, Roh

Critical revision of the manuscript for important intellectual content: Bae, Nam, Roh

Statistical analysis: Bae, Roh

Administrative technical or material support: Roh, Choi, Nam, Kim

Supervision: Roh, Choi, Nam

Funding: No funding was received in support of this work.

\section{Compliance with ethical standards}

Conflict of interest: The authors declare no competing interests.

Ethical approval: This study was approved by the Institutional Review Board of Asan Medical Center, Seoul, Republic of Korea.

Informed consent: Informed consent from each patient was waived.

\section{References}

1. S.A. Hundahl, I.D. Fleming, A.M. Fremgen, H.R. Menck, A National Cancer Data Base report on 53,856 cases of thyroid carcinoma treated in the U.S., 1985-1995 [see commetns]. Cancer 83(12), 26382648 (1998) 
2. J.A. Sipos, E.L. Mazzaferri, Thyroid cancer epidemiology and prognostic variables. Clin. Oncol. (R Coll Radiol) 22(6), 395-404 (2010)

3. K.Y. Bilimoria, D.J. Bentrem, C.Y. Ko, A.K. Stewart, D.P. Winchester, M.S. Talamonti, C. Sturgeon, Extent of surgery affects survival for papillary thyroid cancer, Ann Surg 246(3) (2007) 375 - 81; discussion $381-4$

4. M.A. Adam, J. Pura, L. Gu, M.A. Dinan, D.S. Tyler, S.D. Reed, R. Scheri, S.A. Roman, J.A. Sosa, Extent of surgery for papillary thyroid cancer is not associated with survival: an analysis of 61,775 patients. Ann. Surg. 260(4), 601-605 (2014); discussion 605-7

5. K. Matsuzu, K. Sugino, K. Masudo, M. Nagahama, W. Kitagawa, H. Shibuya, K. Ohkuwa, T. Uruno, A. Suzuki, S. Magoshi, J. Akaishi, C. Masaki, M. Kawano, N. Suganuma, Y. Rino, M. Masuda, K. Kameyama, H. Takami, K. Ito, Thyroid lobectomy for papillary thyroid cancer: long-term follow-up study of 1,088 cases. World J Surg 38(1), 68-79 (2014)

6. B.R. Haugen, E.K. Alexander, K.C. Bible, G.M. Doherty, S.J. Mandel, Y.E. Nikiforov, F. Pacini, G.W. Randolph, A.M. Sawka, M. Schlumberger, K.G. Schuff, S.I. Sherman, J.A. Sosa, D.L. Steward, R.M. Tuttle, L. Wartofsky, 2015 American Thyroid Association Management Guidelines for Adult Patients with Thyroid Nodules and Differentiated Thyroid Cancer: The American Thyroid Association Guidelines Task Force on Thyroid Nodules and Differentiated Thyroid Cancer, Thyroid: official journal of the American Thyroid Association 26(1) (2016) 1-133

7. F.I. Macedo, V.K. Mittal, Total thyroidectomy versus lobectomy as initial operation for small unilateral papillary thyroid carcinoma: A meta-analysis. Surg. Oncol. 24(2), 117-122 (2015)

8. K.J. Kovatch, C.W. Hoban, A.G. Shuman, Thyroid cancer surgery guidelines in an era of de-escalation. Eur. J. Surg. Oncol. 44(3), 297-306 (2018)

9. A. Griffin, J.P. Brito, M. Bahl, J.K. Hoang, Applying Criteria of Active Surveillance to Low-Risk Papillary Thyroid Cancer Over a Decade: How Many Surgeries and Complications Can Be Avoided? Thyroid: official journal of the American Thyroid Association 27(4), 518-523 (2017)

10. B.H. Lang, C.K.H. Wong, Lobectomy is a more Cost-Effective Option than Total Thyroidectomy for 1 to $4 \mathrm{~cm}$ Papillary Thyroid Carcinoma that do not Possess Clinically Recognizable High-Risk Features. Ann Surg Oncol 23(11), 3641-3652 (2016)

11. J. Ryu, Y.M. Ryu, Y.S. Jung, S.J. Kim, Y.J. Lee, E.K. Lee, S.K. Kim, T.S. Kim, T.H. Kim, C.Y. Lee, S.Y. Park, K.W. Chung, Extent of thyroidectomy affects vocal and throat functions: a prospective observational study of lobectomy versus total thyroidectomy. Surgery 154(3), 611-620 (2013)

12. J.L. Roh, J.Y. Park, C.I. Park, Total thyroidectomy plus neck dissection in differentiated papillary thyroid carcinoma patients: pattern of nodal metastasis, morbidity, recurrence, and postoperative levels of serum parathyroid hormone. Ann. Surg. 245(4), 604-610 (2007)

13. C. Cox, M. Bosley, L.B. Southerland, S. Ahmadi, J. Perkins, S. Roman, J.A. Sosa, D. Carneiro-Pla, Lobectomy for treatment of differentiated thyroid cancer: can patients avoid postoperative thyroid hormone supplementation and be compliant with the American Thyroid Association guidelines? Surgery 163(1), 75-80 (2018) 
14. D. Ahn, J.H. Sohn, J.H. Jeon, Hypothyroidism Following Hemithyroidectomy: Incidence, Risk Factors, and Clinical Characteristics. J. Clin. Endocrinol. Metab. 101(4), 1429-1436 (2016)

15. D.S. Cooper, G.M. Doherty, B.R. Haugen, R.T. Kloos, S.L. Lee, S.J. Mandel, E.L. Mazzaferri, B. Mclver, F. Pacini, M. Schlumberger, S.I. Sherman, D.L. Steward, R.M. Tuttle, Revised American Thyroid Association management guidelines for patients with thyroid nodules and differentiated thyroid cancer. Thyroid: official journal of the American Thyroid Association 19(11), 1167-1214 (2009)

16. A.A. Carhill, D.R. Litofsky, D.S. Ross, J. Jonklaas, D.S. Cooper, J.D. Brierley, P.W. Ladenson, K.B. Ain, H.G. Fein, B.R. Haugen, J. Magner, M.C. Skarulis, D.L. Steward, M. Xing, H.R. Maxon, S.I. Sherman, Long-Term Outcomes Following Therapy in Differentiated Thyroid Carcinoma: NTCTCS Registry Analysis 1987-2012, The Journal of clinical endocrinology and metabolism 100(9) (2015) 3270-9

17. Z. Wang, T.E. Angell, W. Sun, Y. Qin, L. He, W. Dong, D. Zhang, T. Zhang, L. Shao, C. Lv, P. Zhang, H. Guan, H. Zhang, Analysis of the strategy of LT4 prescribing and TSH monitoring for thyroid carcinoma after lobectomy. Annals of translational medicine 8(19), 1238 (2020)

18. E.K. Lee, Y.E. Kang, Y.J. Park, B.S. Koo, K.W. Chung, E.J. Ku, H.R. Won, W.S. Yoo, E. Jeon, S.H. Paek, Y.S. Lee, D.M. Lim, Y.J. Suh, H.K. Park, H.J. Kim, B.H. Kim, M. Kim, S.W. Kim, K.H. Yi, S.K. Park, E.J. Jung, J.Y. Choi, J.S. Bae, J.H. Hong, K.H. Nam, Y.K. Lee, H.W. Yu, S. Go, Y.M. Kang, A. Multicenter, Randomized, Controlled Trial for Assessing the Usefulness of Suppressing Thyroid Stimulating Hormone Target Levels after Thyroid Lobectomy in Low to Intermediate Risk Thyroid Cancer Patients (MASTER): A Study Protocol, Endocrinology and metabolism (Seoul, Korea) (2021)

19. L. Lamartina, T. Montesano, R. Falcone, M. Biffoni, G. Grani, M. Maranghi, L. Ciotti, L. Giacomelli, V. Ramundo, C. Lomonaco, C.R. Di Gioia, L. Piernatale, G. Ronga, C. Durante, IS IT WORTH SUPPRESSING TSH IN LOW- AND INTERMEDIATE-RISK PAPILLARY THYROID CANCER PATIENTS BEFORE THE FIRST DISEASE ASSESSMENT?, Endocrine practice: official journal of the American College of Endocrinology and the American Association of Clinical Endocrinologists 25(2), 165-169 (2019)

20. D. Ahn, G.J. Lee, J.H. Sohn, J.H. Jeon, Oncological impact of hypothyroidism and levothyroxine supplementation following hemithyroidectomy in patients with papillary thyroid carcinoma. Head Neck 42(5), 1004-1013 (2020)

21. R. Reed, A. Strumpf, T.G. Martz, K.J. Kavanagh, K.L. Fedder, M.J. Jameson, D.C. Shonka, Jr., 2015 American Thyroid Association guidelines and thyroid-stimulating hormone suppression after thyroid lobectomy. Head Neck 43(2), 639-644 (2021)

22. S.M. Hyun, H.Y. Song, S.Y. Kim, S.Y. Nam, J.L. Roh, M.W. Han, S.H. Choi, Impact of combined prophylactic unilateral central neck dissection and hemithyroidectomy in patients with papillary thyroid microcarcinoma. Ann Surg Oncol 19(2), 591-596 (2012)

23. M.I. Surks, E. Ortiz, G.H. Daniels, C.T. Sawin, N.F. Col, R.H. Cobin, J.A. Franklyn, J.M. Hershman, K.D. Burman, M.A. Denke, C. Gorman, R.S. Cooper, N.J. Weissman, Subclinical thyroid disease: scientific review and guidelines for diagnosis and management, Jama 291(2) (2004) $228-38$ 
24. J.G. Kang, Y.A. Kim, J.E. Choi, S.J. Lee, S.H. Kang, Usefulness of 1-year of thyroid stimulating hormone suppression on additional levothyroxine in patients who underwent hemithyroidectomy with papillary thyroid microcarcinoma. Gland surgery 8(6), 636-643 (2019)

25. H. Verloop, M. Louwerens, J.W. Schoones, J. Kievit, J.W. Smit, O.M. Dekkers, Risk of hypothyroidism following hemithyroidectomy: systematic review and meta-analysis of prognostic studies. J. Clin. Endocrinol. Metab. 97(7), 2243-2255 (2012)

26. S. Park, M.J. Jeon, E. Song, H.S. Oh, M. Kim, H. Kwon, T.Y. Kim, S.J. Hong, Y.K. Shong, W.B. Kim, T.Y. Sung, W.G. Kim, Clinical Features of Early and Late Postoperative Hypothyroidism After Lobectomy. J. Clin. Endocrinol. Metab. 102(4), 1317-1324 (2017)

27. D.Y. Lee, J. Seok, W.J. Jeong, S.H. Ahn, Prediction of thyroid hormone supplementation after thyroid lobectomy. J Surg Res 193(1), 273-278 (2015)

28. H.K. Park, D.W. Kim, T.K. Ha, H.J. Choo, Y.M. Park, S.J. Jung, D.H. Kim, S.K. Bae, Factors associated with postoperative hypothyroidism after lobectomy in papillary thyroid microcarcinoma patients. Endocr Res 40(1), 49-53 (2015)

29. S.J. Stoll, S.C. Pitt, J. Liu, S. Schaefer, R.S. Sippel, H. Chen, Thyroid hormone replacement after thyroid lobectomy. Surgery 146(4), 554-558 (2009); discussion $558-60$

30. E.N. Klein Hesselink, M.S. Klein Hesselink, G.H. de Bock, R.T. Gansevoort, S.J. Bakker, E.J. Vredeveld, A.N. van der Horst-Schrivers, I.C. van der Horst, P.W. Kamphuisen, J.T. Plukker, T.P. Links, J.D. Lefrandt, Long-term cardiovascular mortality in patients with differentiated thyroid carcinoma: an observational study. Journal of clinical oncology: official journal of the American Society of Clinical Oncology 31(32), 4046-4053 (2013)

31. L.Y. Wang, A.W. Smith, F.L. Palmer, R.M. Tuttle, A. Mahrous, I.J. Nixon, S.G. Patel, I. Ganly, J.A. Fagin, L. Boucai, Thyrotropin suppression increases the risk of osteoporosis without decreasing recurrence in ATA low- and intermediate-risk patients with differentiated thyroid carcinoma. Thyroid: official journal of the American Thyroid Association 25(3), 300-307 (2015)

32. M.A. Adam, J. Pura, P. Goffredo, M.A. Dinan, T. Hyslop, S.D. Reed, R.P. Scheri, S.A. Roman, J.A. Sosa, Impact of extent of surgery on survival for papillary thyroid cancer patients younger than 45 years. J. Clin. Endocrinol. Metab. 100(1), 115-121 (2015)

33. Y.M. Park, D.Y. Lee, K.H. Oh, J.G. Cho, S.K. Baek, S.Y. Kwon, K.Y. Jung, J.S. Woo, Clinical implications of pathologic factors after thyroid lobectomy in patients with papillary thyroid carcinoma. Oral Oncol 75, 1-5 (2017)

\section{Figures}


A

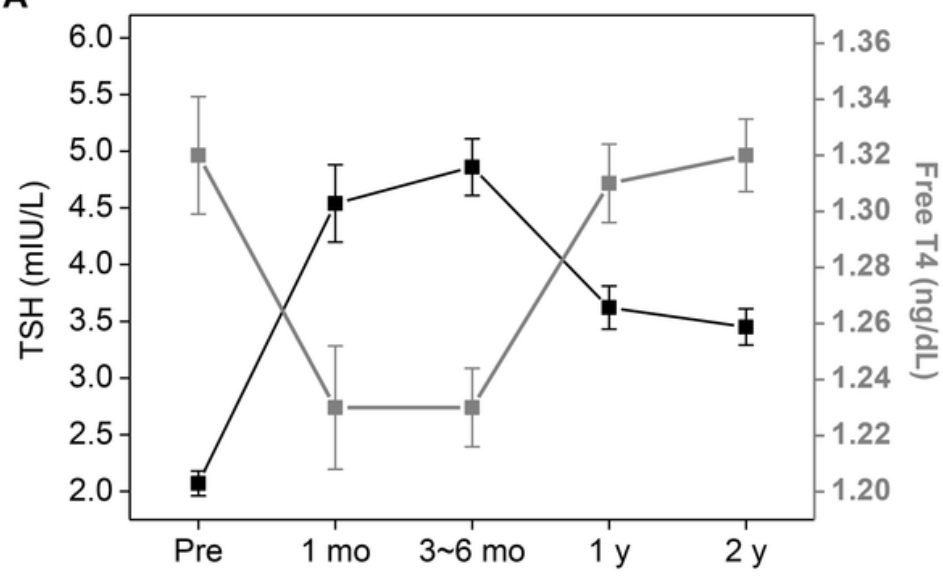

B

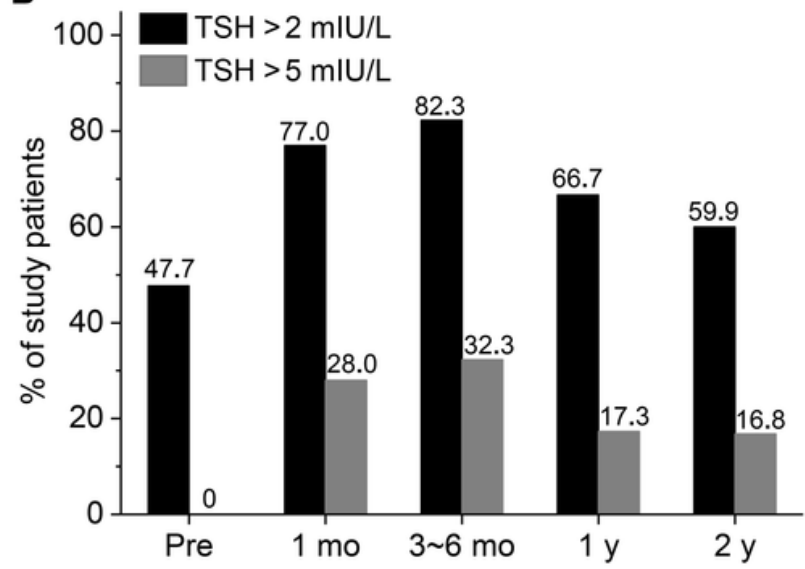

Figure 1

The changes of TSH and free T4 serum concentrations and proportions with high TSH levels in patients who underwent thyroid lobectomy. (A) The dual Y-axis plot of TSH and free T4 serum concentrations following lobectomy. Data are means and standard errors. (B) The proportions of patients with high TSH levels of $>2$ or $>5 \mathrm{mIU} / \mathrm{L}$. Serum TSH and free T4 concentrations and high TSH proportions were calculated for patients who were not on T4 supplementation at the time that thyroid function tests were obtained.

\section{The study cohort: 369 patients}

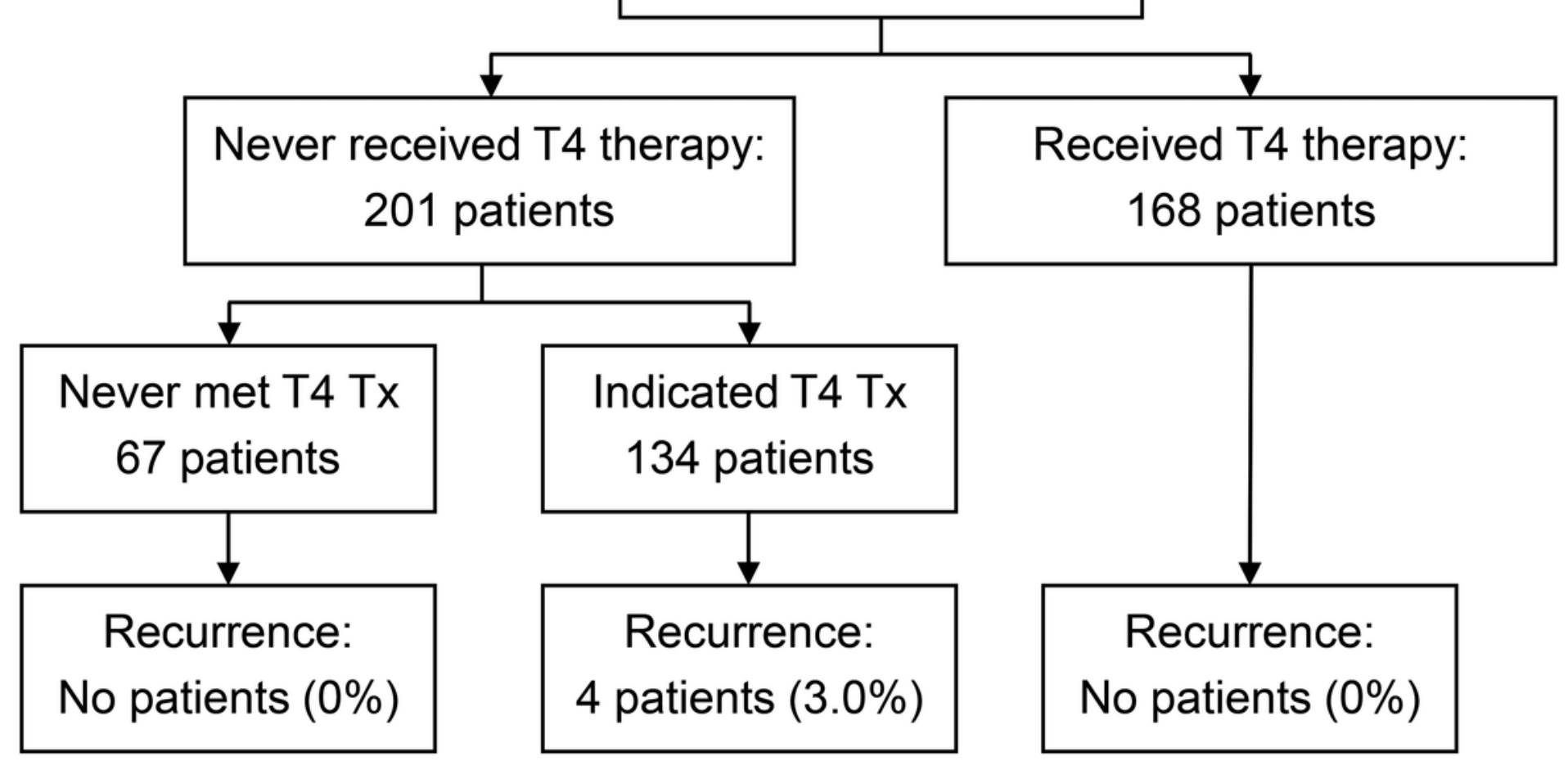

Figure 2 
Flow chart of the study cohort who underwent thyroid lobectomy with or without post-lobectomy TSH suppression for unilateral papillary thyroid carcinoma. TSH stands for thyroid-stimulating hormone; T4 Tx, levothyroxine supplementation therapy. 